\title{
ORIGINAL
}

\section{ANEMIA ASOCIADA A INFECCIÓN POR HELICOBACTER PYLORI EN ESTUDIANTES UNIVERSITARIOS.}

\author{
ANEMIA ASSOCIATED WITH HELICOBACTER PYLORI INFECTION IN \\ UNIVERSITY STUDENTS.
}

Juan Marco Quispe Reyes ${ }^{1}$, Sheein Estephanie Maria Vega Rojas ${ }^{1}$, Isaac kenyi Huayta Quispe ${ }^{1}$, Vasti Evelyn Díaz Quiquiaํㅜㄹ. Dr. Pavel Illich Chávez Cáceres².

\section{RESUMEN}

INTRODUCCIÓN: La anemia se considera una entidad de mayor prevalencia en países en vías de desarrollo, teniendo como causas principales la falta de ingesta nutricional: hierro, ácido fólico, vitamina B12. La anemia ferropénica es la causa más frecuente y afecta en su mayoría a mujeres en edad fértil como en la postmenopausia. Además se ha asociado a Helicobacter pylori, como factor de riesgo para anemia ferropénica.

Objetivo. Determinar la asociación que existe entre la anemia y Helicobacter pylori como factor de riesgo en los estudiantes de la Universidad Peruana Unión en el año 2014.

MATERIALES Y METODO: Estudio de casos y controles. Este trabajo se llevó a cabo en estudiantes de la Universidad Peruana Unión. Se obtuvo la base de datos de estudiantes atendidos en la Clínica Good Hope, con los diagnósticos de anemia ferropénica, seleccionados por medio de un muestreo aleatorio simple determinando el grupo caso de 40 personas con anemia $(n=40)$ y 80 personas sin diagnóstico de anemia siendo el grupo control. Se aplicó una encuesta con las variables de interés para la descripción de la población y el análisis de muestra sanguínea para el hematocrito, hemoglobina (en 48 personas para confirmar los casos y controles) y la prueba rápida antiHelicobacter pylori.

RESULTADOS: Se determinó un odds ratio entre anemia y Helicobacter pylori de 3,8; un chi cuadrado de 10,12 y una $p=0,001$. Este resultado rechaza la hipótesis nula de independencia de las variables y se comprueba la asociación antes propuesta.

DISCUSIÓN: Helicobacter pylori es un factor de riesgo asociado a la anemia ferropénica, aunque se requiere más estudios en nuestra población.

Conclusiones. Helicobacter pylori es un factor de riesgo asociado con anemia, aunque se requieren más estudios en la población de esta investigación.

\section{ABSTRACT}

INTRODUCTION: Anemia is considered an entity of greater prevalence in developing countries, having as main causes the lack of nutritional intake: iron, folic acid, vitamin B12. Iron deficiency anemia is the most frequent cause and affects mostly women of childbearing age as in postmenopause. It has also been associated with Helicobacter pylori as a risk factor for iron deficiency anemia.

Objective. To determine the association that exists between anemia and Helicobacter pylori as a risk factor in the students of the Peruvian Unión University in 2014.

MATERIAL AND METHOD: Cases and controls study. This work was carried out on students of the Union Peruvian University. The database of students attended at the Good Hope Clinic was obtained, with the diagnoses of irondeficiency anemia, selected by means of a simple random sampling, determining the group of 40 people with anemia $(n=40)$ and 80 people without diagnosis of anemia being the control group. A survey was applied with the variables of interest for the description of the population and the blood sample analysis for the hematocrit, hemoglobin (in 48 people to confirm the cases and controls) and the rapid anti-Helicobacter pylori test.

RESULTS: An odds ratio between anemia and Helicobacter pylori of 38 was determined; a square chi of 10.12 and a $p=0.001$. This result rejects the null hypothesis of independence of the variables and the previously proposed association is checked.

DISCUSSION: Helicobacter pylori is a risk factor associated with iron deficiency anemia, although more studies are required in our population.

Conclusions Helicobacter pylori is a risk factor associated with anemia, although more studies are required in the population of this research.

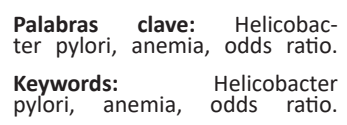

Procedencia y arbitraje: no comisionado, sometido a
arbitraje

Recibido para publicación: 1 de abril 2017

Aceptado para publicación:

15 de diciembre 2017

Citar como:

Rev Cient Cienc Med.

2017; 20(2): 21-25. 


\section{INTRODUCCIÓN}

La anemia es una de las entidades que más prevalencia ha tenido, afectando a la población mundial con $24,8 \%$, siendo su mayor índice de afectados en países en vías de desarrollo, especialmente sudamericanos ${ }^{1}$. La anemia se define como una disminución en la concentración sérica de hemoglobina $(\mathrm{Hb})$. Se ha estudiado la etiología de esta entidad, de las cuales una de las principales causas es la falta de ingesta nutricional, dentro de ellas: hierro, ácido fólico, vitamina $\mathrm{B} 12^{2,3}$. El desequilibrio entre el aporte del hierro y su metabolismo conducen a una anemia ferropénica ${ }^{4}$. La ferropenia es la causa más frecuente de anemia, afectando tanto a mujeres y varones de las diferentes edades. Datos epidemiológicos demuestran la presencia de anemia ferropénica en $2 \%$ de los hombres y en $5 \%$ de las mujeres postmenopáusicas en el mundo desarrollado. En el caso de las mujeres en edad fértil afecta a un $10 \%$ de la población ${ }^{5}$.

Desde muchos años atrás se ha estudiado los factores de riesgo asociados a dicha enfermedad. No fue hasta la última década que apareció una nueva variable: Helicobacter pylori (Hp). El $\mathrm{Hp}$ es la causa más común de gastritis y está asociado a ulcera duodenal ${ }^{6}$. Estudios recientes realizados en poblaciones latinoamericanas han demostrado relación de esta entidad con la anemia ferropénica, tanto en niños como en adolescentes $^{7,8}$. La infección por $\mathrm{Hp}$ induce el secuestro de hierro por diferentes proteínas de unión y transportadores tales como la lactoferrina y la proteína Feo $\mathrm{B}$ incrementando la pérdida de sangre gastrointestinal y/o reduciendo la absorción de hierro secundaria a una gastritis crónica ${ }^{9}$. El Hp genera una respuesta inflamatoria la cual estimula la secreción de hepcidina, regulador del hierro, el cual va a inducir la destrucción lisosomal de los transportadores de hierro impidiendo así su absorción ${ }^{7}$. El Hp disminuye la producción de ácido ascórbico, el facilitador de la conversión de hierro férrico a hierro ferroso, disminuyendo su absorción intestinal ${ }^{10}$.

El propósito de este estudio es medir el odds ratio (OR) de desarrollar la anemia al hecho de estar expuesto al Hp en un grupo de estudiantes de la Universidad Peruana Unión, para determinar si la evidencia teórica se correlaciona con los hallazgos epidemiológicos ${ }^{11}$.

\section{MATERIALES Y MÉTODOS}

Estudio de casos y controles. Realizado entre noviembre y diciembre del 2014 en la Universidad
Peruana Unión, Ñaña, Perú. De la población total de estudiantes con diagnóstico de anemia ( $\mathrm{N}=643$ estudiantes); se seleccionó a 40 estudiantes, entre 16 - 35 años de edad; y 75 personas para los controles de las personas sin diagnóstico de anemia, para el estudio de Helicobacter pylori; mediante un muestreo aleatorio simple; El tamaño muestral fue calculado para un nivel de confianza del $95 \%$, un poder estadístico de $80 \%$, con una frecuencia de exposición presuntiva de $55 \%$.

Del total de estudiantes que participaron de la extracción de muestra sanguínea en el laboratorio se comprobaron los datos de Hemoglobina y Hematocrito, de un $41,7 \%$ de la población mientras que al 58,3\% sus datos fueron basados en resultados obtenidos meses anteriores en el laboratorio de la universidad peruana unión.

Se realizó un cuestionario que recolecta variables de interés tales como edad, sexo y procedencia.

Laboratorio: Los análisis fueron obtenidos por extracción de sangre. Después de la extracción se le realizaron estudios para determinar la hemoglobina, hematocrito, y la prueba rápida anti-Hp las cuales cuentan con una sensibilidad entre $88-95 \%$ y especificidad de $86-95 \%$ realizadas en el laboratorio de la Universidad Peruana Unión (Ñaña, Perú).

Métodos de Análisis: para determinar los rangos de inclusión en estudiantes con anemia se puso los parámetros según la OMS ( $<12$ en mujeres; $<13$ en hombres). Anemia leve en mujeres $\mathrm{Hb}$ entre 11,0 y $11,9 \mathrm{~g} / \mathrm{dl}$ y anemia moderada de $8 \mathrm{y}$ $10,9 \mathrm{~g} / \mathrm{dl}$. En los casos masculinos se consideró anemia leve a una $\mathrm{Hb}$ entre 10 y $12,9 \mathrm{~g} / \mathrm{dl} \mathrm{y}$ anemia moderada $\mathrm{Hb}$ entre 8 y 10,9 g/dl. El grupo control femenino se constituyó de estudiantes con una $\mathrm{Hb}$ superior o igual a $12 \mathrm{~g} / \mathrm{dl}$; el grupo control masculino con $\mathrm{Hb}$ superior o igual a $13 \mathrm{~g} / \mathrm{dl}$. Se excluyeron los casos y controles que no respondieron la totalidad de la encuesta, estudiantes retirados o que pertenecieran a otra universidad.

\section{Análisis Estadísticos}

Las asociaciones de la encuesta y los valores independientes hallados en el laboratorio relatan la prevalencia de $\mathrm{Hp}$ en pacientes que tienen anemia. Los datos obtenidos fueron registrados en una base de datos para el análisis estadístico con el programa Microsoft Excel® 7.0 en la primera fase y luego se usó el software SPSS versión 22.0 para la fase 2 .

Se realizó el presente estudio respetando las normas éticas de justicia, autonomía y no maleficencia. 


\section{RESULTADOS}

Se encontró en 63 personas infección por Hp, dentro de los cuales 30 pertenecen al grupo de casos y 33 al grupo control (ver tabla 1). El intervalo de confianza en de $95 \%$ con valores de 1,$57 ; 1,31 ; 2,11$ y 2,45 para edad, sexo, procedencia y H. Pylori respectivamente. El error estándar en las variables fue menor o igual a 0,095. Para estimar el riesgo se utilizó la función Odds ratio con un valor de 3,818; grado de libertad igual a 1 y un valor "p" de 0,001 , lo cual rechaza la hipótesis nula de independencia de las variables y se comprueba la asociación antes propuesta (ver tabla 2).

Tabla 1. Descripción de la población expuesta.

\begin{tabular}{|c|c|c|c|c|c|c|c|}
\hline & ANEMIA & & & \multicolumn{2}{|c|}{ NO ANEMIA } & \multirow{3}{*}{$\begin{array}{l}\text { Intervalo } \\
\text { de } \\
\text { confianza } \\
(95 \%)\end{array}$} & \multirow{3}{*}{$\begin{array}{l}\text { Error } \\
\text { estándar }\end{array}$} \\
\hline & \multicolumn{2}{|l|}{ Casos } & \multicolumn{2}{|c|}{ Controles } & & & \\
\hline & $N$ & $\%$ & $\mathrm{~N}$ & $\%$ & & & \\
\hline \multirow{4}{*}{ 喈 } & $\begin{array}{l}\text { Adolecentes 16- } \\
19\end{array}$ & 22 & 55 & 36 & 48 & \multirow[t]{4}{*}{1,57} & \multirow[t]{4}{*}{,058 } \\
\hline & $\begin{array}{l}\text { Juventud } \\
\text { temprana } \\
20-24\end{array}$ & 14 & 35 & 35 & 47 & & \\
\hline & $\begin{array}{l}\text { Juventud tardía } \\
25-35\end{array}$ & 4 & 10 & 4 & 5 & & \\
\hline & Total & 40 & 100 & 75 & 100 & & \\
\hline \multirow{3}{*}{$\begin{array}{l}0 \\
\text { ய } \\
\text { ஸे }\end{array}$} & Femenino & 38 & 95 & 41 & 55 & \multirow[t]{3}{*}{1,31} & \multirow[t]{3}{*}{,043 } \\
\hline & Masculino & 2 & 5 & 34 & 45 & & \\
\hline & Total & 40 & 100 & 75 & 100 & & \\
\hline \multirow{6}{*}{ 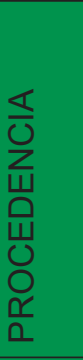 } & $\begin{array}{l}\text { Lima- } \\
\text { metropolitana }\end{array}$ & 15 & 37,5 & 28 & 37,34 & \multirow[t]{6}{*}{2,11} & \multirow[t]{6}{*}{,095 } \\
\hline & Resto costa & 9 & 22,5 & 17 & 22,67 & & \\
\hline & Sierra & 13 & 32,5 & 24 & 32 & & \\
\hline & Selva & 3 & 7,5 & 5 & 6,66 & & \\
\hline & Extranjeros & 0 & 0 & 1 & 1.33 & & \\
\hline & Total & 40 & 100 & 75 & 100 & & \\
\hline \multirow{3}{*}{ 茫艺 } & $\mathrm{Si}$ & 30 & 75 & 33 & 44 & \multirow[t]{3}{*}{2,45} & \multirow[t]{3}{*}{047} \\
\hline & No & 10 & 25 & 42 & 56 & & \\
\hline & Total & 40 & 100 & 75 & 100 & & \\
\hline
\end{tabular}

\section{DISCUSIÓN}

En este estudio se tiene un OR de 4,2 (ver tabla 2), el Hp es un factor importante para que se pueda producir la anemia, un estudio sobre el tema expone que Helicobacter pylori causa

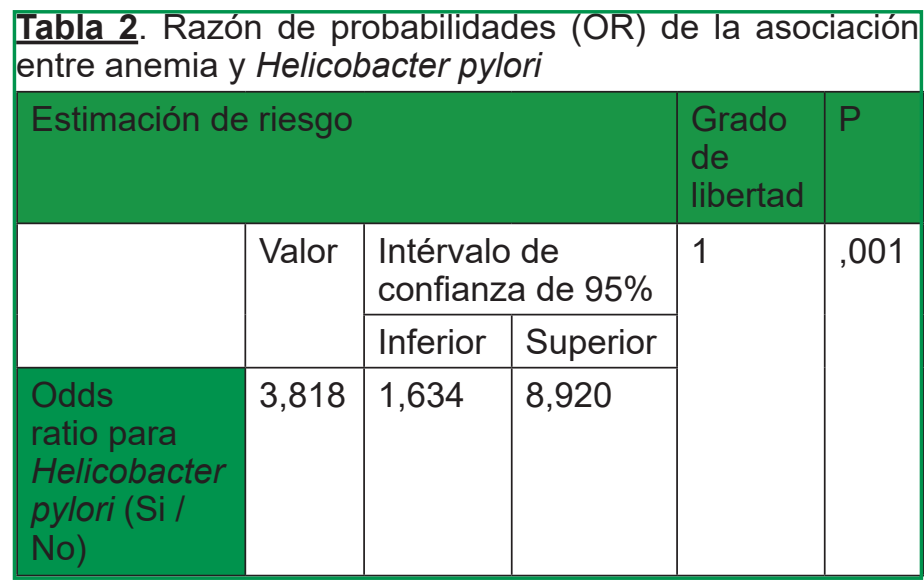

enfermedades gastroduodenales como gastritis crónica asintomática en un $85 \%$ de los casos, seguido por ulcera duodenal y por último de adenocarcinoma gástrico, pero también produce 
enfermedades extra gástricas y hace mención a la anemia por deficiencia de hierro ${ }^{7}$.

Se ha demostrado que la absorción del hierro esta alterada por incremento de la hepcidina causada por la inflamación gástrica, los niveles de lactoferrina son altos perdiéndose hierro, esto es producido por los neutrófilos y por la alteración del $\mathrm{pH}^{7,16}$. La alteración del $\mathrm{pH}$, a causa de inflamación producida por el $\mathrm{Hp}$, no permite que el hierro se pueda reducir de hierro férrico a hierro ferroso, todo esto se suma a la disminución de los niveles de ácido ascórbico ${ }^{21,22}$. El hierro también es importante para el $\mathrm{Hp}$, por lo tanto hay competencia en cuanto el hospedero y el Hp para la utilización del hierro ${ }^{14}$.

En un estudio de tipo transversal no se encontró asociación entre $\mathrm{Hp}$, pero exponen que se requieren más estudios para demostrar la falta de asociación, mostrando también las limitaciones del estudio que se llevó a cabo en Brasil $^{12}$. En otro estudio, realizado en niños entre 6 y 12 años se ve que existe relación entre Hp y la deficiencia de hierro, en la que aconsejan no solo tratar la anemia, sino que también es necesaria la eliminación de esta bacteria ${ }^{13}$.

Estudios indican que las enfermedades hematológicas están producidas por $\mathrm{Hp}$, entre estas se señalan púrpura trombocitopénica idiopática, déficit de cobalamina y anemia ferropénica, la que tiene evidencia comparativa con $\mathrm{Hp}$ es la anemia ferropénica ${ }^{14}$. Sin embargo en contexto del presente estudio, el factor genético es un factor de riesgo para padecer anemia ferropénica (tabla 1).

En otros países, desde 1991, existen reportes de casos en los que después de eliminar el Hp se da resolución a la anemia ferropénica. Se menciona que en estudios epidemiológicos no se puede diferenciar si los niveles bajos de ferritina se deben por ser seropositivos a Hp o por otras causas que explicarían la deficiencia de hierro ${ }^{15}$.

En otro estudio se concluye que la eliminación del Hp ayuda a una mejor respuesta al tratamiento para anemia ferropénica ${ }^{17}$. Otro estudio explica la relación de Hp y anemia por deficiencia de hierro, éste muestra que existe clínicamente una significancia entre $\mathrm{Hp}$ y anemia por deficiencia de hierro ${ }^{18}$.

En este estudio tuvo como método de diagnóstico de infección por Hp mediante una prueba rápida de anti-Helicobacter pylori, esta prueba es recomendada por ser un método no invasivo que mide de forma indirecta la presencia de Hp; esta además de tener muy pocas complicaciones fue la más adecuada para este trabajo $^{19}$ ya que se evita los el uso de métodos directos invasivos para diagnóstico de $\mathrm{Hp}$, pues además de posibles complicaciones, son más $\operatorname{costosos}^{20}$. En la clínica la forma más eficiente de detección de Hp por lo ya mencionado.

El estudio trató de determinar la asociación entre Hp con anemia, en relación a la variable anemia, sin embargo vale aclarar no se tiene el diagnostico específico entre los diferentes tipos de anemia, por lo tanto es una de la limitaciones que tiene el trabajo ya que la evidencia científica del Hp se asocia más con anemia ferropénica ${ }^{14,15,16}$. También se tiene en cuenta que la muestra fue obtenida de modo aleatorio simple, por lo que los casos y controles no fueron homogéneos en cuanto a características, además que se evidencia poca cantidad de varones con una cantidad predominante en mujeres.

En un estudio prospectivo encontraron que la infección por Hp se consideró la causa de la anemia cuando se observó una resolución completa de la anemia sin suplementos de hierro después de la erradicación, la cual fue evaluada a los 6 y 12 meses del seguimiento. Se concluyó que la infección por Hp gástrico es una causa frecuente de anemia refractaria o dependiente de hierro de origen previamente desconocido en pacientes adultos ${ }^{23}$.

Por otro lado recientemente se realizó un estudio retrospectivo para explorar la asociación entre la infección por H. pylori y la anemia en la población china, donde se hallaron datos similares a los de la presente investigación, ya que el nivel de $\mathrm{Hb}$ fue menor en el grupo $\mathrm{Hp}(+)$ que en el grupo Hp (-). Este estudio indica que la infección por $\mathrm{Hp}$ puede estar relacionada con la anemia y el nivel de $\mathrm{Hb}$ en la población china ${ }^{24}$.

La anemia es un problema a nivel mundial por lo tanto la determinación de Hp como factor de riesgo ayudaría a reducir este problema si se detecta y se da un correcto tratamiento. Teniendo en cuenta que la prevalencia de Hp es muy alta.

Los diversos estudios demuestran que existe asociación de Hp como factor de riesgo para la anemia. Es necesario determinar el tipo específico de anemia para ver una relación concreta entre $\mathrm{Hp}$ y el tipo de anemia por lo que se requieren otros estudios.

\section{AGRADECIMIENTO}

Se agradece al Mg. Benjamín Jarama, Mg. Pool Marcos Carbajal, Ing. Félix Palacios, compañeros de clase y a la Escuela de Medicina Humana de la Universidad Peruana Unión. 


\section{REFERENCIAS}

1.World Health Organization. Prevalencia mundial de la anemia y número de personas afectadas; 2015. Acceso 14 de abril 2015. Disponible en: http:// www.who.int/vmnis/database/anaemia/anaemia_data_status_t2/es/

2.Fernández N, Aguirrezabalaga B. Anemias en la infancia. Anemia ferropénica; Bol Pediatr;2006: 46: 311-7. Disponible en: https://www.sccalp. org/boletin/46_supl2/BolPediatr2006_46_supl2_311-317.pdf

3.Bichara F, Amancio O, Gutiérrez-Salmean G, Fuchs V. DETERMINACIÓN DEL TIPO DE ANEMIA Y SU RELACIÓN CON LA INGESTIÓN ALIMENTARIA Y MARCADORES BIOQUÍMICOS EN PACIENTES CON CÁNCER CÉRVICO UTERINO; Rev Chil Nutr;2009;36(4):1074-9.Disponible en: https://scielo.conicyt.cl/pdf/rchnut/v36n4/art03.pdf

4.Suárez JL. Reyes GC. Herreros L. Helicobacter pylori: Revisión de los aspectos fisiológicos y patológicos;MÉD.UIS;2011;24(3):287296.Disponible en: http://www.scielo.org.co/scielo.php?script=sci_ arttext\&pid=S0121-03192011000300006

5.Serrano C, Villagrán A, Harris P. Helicobacter pylori: una causa no tradicional de deficiencia de hierro y anemia;Rev Chil Pediatr;2012;83 (1):13-23.Disponible en: https://scielo.conicyt.cl/scielo.php?script=sci_ arttext\&pid=S0370-41062012000100002

6.Cabrera TV, Jiménez M. Sulfato ferroso y preeclampsia. Estudio de casos y controles realizado en dos hospitales de Bogotá. Rev. TEORIA Y PRAXIS INVESTIGATIVA.2010; 5(2); 43 - 51.Disponible en: https://dialnet. unirioja.es/descarga/articulo/3702396.pdf

7.Pellicano R, Franceschi F, Saracco G, Fagoonee S, Roccarina D. and Gasbarrini A. Helicobacter pylori and Extragastric Diseases. Helicobacter. 2014; 19(1): 58-68.Disponible en: http://onlinelibrary.wiley.com/doi/10.1111/ j.1523-5378.2009.00699.x/abstract

8.Melendez M. Revisión narrativa sobre la infección de Helicobacter pylori y la anemia ferropénica en niños de 1 a 17 años. [Tesis para Título Profesional]. Bogotá: Pontificia Universidad Javeriana; 2010.

9.Husson MO, Legrand D, Spik G, Leclerc H. Iron Acquisition by Helicobacter pylori: Importance of Human Lactoferrin;Infect Inmun;1993;61(6):2694-7. Disponible en: https://www.ncbi.nlm.nih.gov/pmc/articles/PMC280902/

10.Banerjee S, Hawksby C, Miller S, Beattie AD, McColl Ke. Effect of Helicobacter pyloriand its eradication ascorbic acid;Gut;1994; 35:317-22. Disponible en: http://gut.bmj.com/content/gutjnl/35/3/317.full.pdf

11. Cruz $F$, Ortiz E, León R. Relación entre la anemia ferropénica y la infección por Helicobacter pylori. Rev Cub Hem Inm y Med Tr;2007; 23 (2). Disponible en: http://www.bvs.sld.cu/revistas/hih/vol23_2_07/hih03207.html

12.Santos IS, Costa G, Valle NC et al. Helicobacter pylori and anemia: a community-based cross-sectional study among adults in Southern Brazil;Cad. Saúde Pública;2009;25(12): 2653-2660.Disponible en: http:// www.scielo.br/pdf/csp/v25n12/12.pdf

13.Ruiz-Álvarez V, Reboso-Pérez J, Hernández-Triana M. Asociación entre la infección por Helicobacter pylori y anemia en niños de edad escolar,Rev Cubana Invest Bioméd;2005;24(2).Disponible en: http://scielo. sld.cu/pdf/ibi/v24n2/ibi02205.pdf

14.Urrego J, Otero W, Gómez M. Helicobacter pylori y enfermedades hematológicas;Rev Col Gastroenterol;2013;28(4):329 - 337.Disponible en: http://www.redalyc.org/pdf/3377/337731611008.pdf
15.Suja D. David J. Iron-deficiency anemia and Helicobacter pylori infection: A review of the evidence;Am J Gastroenterol;2005;100(2):453-459.Disponible en: https://www.ncbi.nlm.nih.gov/pubmed/15667507

16.Yon H, Yoo J, Na G, Ichiro I, Yukiriko A, Nagahito T, Esteban C. Lactoferrin sequestration and its contribution to iron-deficiency anemia in Helicobacter pylori-infected gastric mucosa;J Gastroenterol Hepatol;2003;18(8):980-985.Disponible en: https://www.ncbi.nlm.nih.gov/ pubmed/12859729

17.Choe YH, Kim SK, Son BK, Lee DH, Hong Y, Pai S. Randomized placebo-controlled trial of Helicobacter pylori eradication for iron-deficiency anemia in preadolescent children and Adolescents; Helicobacter:1999;4(2):135-9.

18.Rabieipoor M, Maleki D, Kazemzade J, Rabieipoor S, Behrozian R, \& Agdashi M. The study of relationship between helicobacter pylori and Iron Deficiency Anemia in patient who referred to hematological clinic in Educational Emam Khomeini hospital;Annals of Biological Research;2011;2(5):449-454.Disponible en: http://docplayer.net/38632974Scholars-research-library-annals-of-biological-research-2011-2-5-httpscholarsresearchlibrary-com-archive-html-issn.html

19.Öztürk E. Diagnostic methods of Helicobacter pylori, infection; Gülhane Týp Dergisi;2008;50:60-64.Disponible en: http://uvt.ulakbim.gov.tr/ uvt/index.

20.Gatta L., Ricci C, TampieriA, and Vaira D. Non-invasive techniques for the diagnosis of Helicobacter pylori infection; Clin Microbiol Infect;2003;9(6): 489-96. Disponible en: http://www.clinicalmicrobiologyandinfection.com/ article/S1198-743X(14)63998-7/fulltext

21.Chuang C, Sheu B, Yang H, Lee S, Kao A, Cheng $H$, Chang W \& Yao W. Ghrelin and Leptin Gender Difference of Circulating Ghrelin and Leptin Concentrations in Chronic Helicobacter pylori Infection;Journal compilation Blackwell Publishing Ltd;Helicobacter;2009;14:54-60.

22.González MA, Olivares J. The gene frpB2 of Helicobacter pylori encodes and hemoglobin-binding protein involved in iron acquisition; Springer Science Business Media, LLC;2009;22(6):889-94.Disponible en: http://europepmc.org/abstract/med/19357969

23.Monzón H, Forné M, Esteve M, Rosinach M, Loras C, Espinós J, et al. Helicobacter pylori infection as a cause of iron deficiency anaemia of unknown origin;World. J Gastroenterol; 2013;19(26):4166-4171. Disponible en: https://www.ncbi.nlm.nih. gov/pmc/articles/PMC3710418/pdf/WJG-19-4166.pdf

24.Mei-Yan X, Bing C, Bao-Shi Y, Jian Y, Lan L, Qing-Bin L. Association of anaemia with Helicobacter pylori infection: a retrospective study. Sci Rep; 2017;7:13434. Disponible en: https://www.nature.com/articles/s41598-017-13955-3GastroGastroenter ol;2013;19(26):4166-4171. Disponible en: https://www.ncbi.nlm.nih.gov/pmc/ articles/PMC3710418/pdf/WJG-19-4166.pdf

25. Mei-Yan Xu, Bing Cao, Bao-Shi Yuan, Jian Yin, Lan Liu, Qing-Bin Lu. Association of anaemia with Helicobacter pylori infection: a retrospective study. Sci Rep. 2017; 7: 13434. Published online 2017 Oct 18. doi: 10.1038/s41598-017-13955-3C 